\title{
ON A THEOREM OF R. MOUFANG
}

\author{
R. H. BRUCK
}

A loop is a system with a binary operation, possessing a unit 1 , and such that any two of the elements in the equation $x y=z$ uniquely determine the third. A Moufang loop [1, chap. 2 ${ }^{1}$ may be characterized by the tidentity $x y \cdot z x=(x \cdot y z) x$. The following theorem is due to R. Moufang [2].

Theorem. If $a b \cdot c=a \cdot b c$ for three elements $a, b, c$ of $a$ Moufang loop, the subloop generated by them is associative.

We give a particularly simple proof for the commutative case. (This proof, although complete in itself, stems from the theory of autotopisms introduced in [1], which will be applied elsewhere to the noncommutative case.) Henceforth let $G$ be a commutative Moufang loop. For each $x$ in $G$ define the permutation $R(x)$ by $y R(x)$ $=y x$. The defining relation can be written in the two forms

$$
y x \cdot z x=(y z \cdot x) x, \quad y R(x) \cdot z R(x)=(y z) R(x)^{2} .
$$

If we take $z=x$ in (1), $y x \cdot x x=(y x \cdot x) x$. If we replace $y x$ by $y$,

$$
y \cdot x x=y x \cdot x .
$$

If $x^{-1}$ is defined by $x x^{-1}=1$ (so that $\left(x^{-1}\right)^{-1}=x$ ), (1) with $z=x^{-1}$ gives $y x=\left(y x^{-1} \cdot x\right) x, y=y x^{-1} \cdot x$ and

$$
y x \cdot x^{-1}=y, \quad R(x)^{-1}=R\left(x^{-1}\right) .
$$

Let \&s be the group generated by the $R(x)$, and consider its elements $S=R\left(a_{1}\right) R\left(a_{2}\right) \cdots R\left(a_{n}\right), \quad T=R\left(a_{1}\right)^{2} R\left(a_{2}\right)^{2} \cdots R\left(a_{n}\right)^{2}$. By (3), every element of (B) can be put in the form $S$. By repeated application of (1), $y S \cdot z S=(y z) T$. If the $a_{i}$ are chosen so that $1 S=1$, let $y=1$ and have $S=T$. Thus the subgroup $\Im$ of $\&$, consisting of the $S$ with $1 S=1$, is a group of automorphisms of $G$. We use this "remark" several times; its value lies in the readily verified fact that the elements left invariant by a set of automorphisms of a loop form a subloop.

Let $H$ be the subloop of the theorem and $H_{1}$ the subset consisting of the $z$ in $H$ such that $a b \cdot z=a \cdot b z$. Equivalently, $z S=z$ where $S$ $=R(a b) R\left(a^{-1}\right) R\left(b^{-1}\right)$. By the remark, $S$ induces an automorphism of $H$, so $H_{1}$ is a subloop of $H$. Moreover $H_{1}$ contains $c$, by hypothesis,

Received by the editors December 1, 1949.

1 Numbers in brackets refer to the references cited at the end of the paper. 
and $a, b$, by (2). Hence $H_{1}=H$.

In particular, therefore, $a b \cdot c^{-1}=a \cdot b c^{-1}$. If we apply (3) and (1) in turn, $a b=\left(a \cdot b c^{-1}\right) c, a b \cdot c=a c \cdot b$. It is now easy to see that the relation $a b \cdot c=a \cdot b c$ remains true under all permutations of $a, b, c$. We deduce among other things that $a c \cdot z=a \cdot c z$ for all $z$ in $H$.

Let $H_{2}$ be the subset consisting of the $y$ in $H$ such that $a y \cdot z=a \cdot y z$ for all $z$ in $H$. By the remark, $H_{2}$ is a subloop of $H$, containing $a$, by (2), and $b, c$, by the above proofs. Hence $H_{2}=H$.

A similar argument now gives $x y \cdot z=x \cdot y z$ for all $x, y, z$ in $H$.

\section{REFERENCES}

1. R. H. Bruck, Contributions to the theory of loops, Trans. Amer. Math. Soc. vol. 60 (1946) pp. 245-354.

2. Ruth Moufang, Zur Struktur von Alternativkörpern, Math. Ann. vol. 110 (1935) pp. 416-430.

UNIVERSITY OF WISCONSIN 